\title{
Conocer mejor a nuestros alumnos en beneficio de nuestras acciones educativas
}

\author{
Knowing better our students to improve our educational actions
}

\author{
Luis E. Prieto-Marín ${ }^{a}$, Ana M. Rueda-Salazar ${ }^{b}$
}

\begin{abstract}
:
This paper presents some results from two research projects from the Language Learning Center at Acatlán campus of the National University of Mexico (UNAM). The main objective was to get direct knowledge about our students, their representations of a foreign language, their motivations and their metacognitive perception of their learning process through diverse tasks. The main recommendations derived from these studies are presented.
\end{abstract}

\section{Keywords:}

Learning, foreign language, competence, action competence, task learning, representations, intercultural

\section{Resumen:}

Se presentan algunos resultados de dos investigaciones realizadas en el Centro de Enseñanza de Idiomas de la Facultad de Estudios Superiores Acatlán de la Universidad Nacional Autónoma de México. El objetivo principal era conocer directamente de los alumnos sus representaciones sobre lo que es una lengua extranjera, sus motivaciones para aprenderla y la percepción metacognitiva de su propio aprendizaje a través de diversas tareas. Se presentan las principales recomendaciones derivadas de estos estudios

\section{Palabras Clave:}

Enseñanza, idiomas, competencias, competencia de acción, aprendizaje por tareas, representaciones, intercultural

\section{INTRODUCCIÓN}

En el presente artículo se presentan algunos resultados de dos investigaciones realizadas en el Departamento de Francés del Centro de Enseñanza de Idiomas, en la Facultad de Estudios Superiores Acatlán de la Universidad Nacional Autónoma de México. Ana María Rueda llevó a cabo un estudio sobre la Enseñanza del francés mediante proyectos no centrados en el lenguaje, en el marco de un trabajo de posgrado. Su objetivo era conocer a través de encuestas la percepción de los propios alumnos sobre su motivación y aprovechamiento con la realización de actividades complementarias no centradas en el lenguaje. Por su parte, Luis E. Prieto realizó otra investigación sobre el Concepto y percepción de la lengua extranjera y sus implicaciones en maestros y alumnos del CEI Acatlán, una investigación de tipo cualitativo mediante entrevistas. Ambos estudios se llevaron a cabo durante el año escolar 2018-2019. Las dos investigaciones se comentan aquí por referirse a la misma institución, a los mismos actores y al mismo contexto específico.

Como uno de los antecedentes directos de estos estudios destinados a conocer a nuestros alumnos para adecuar la enseñanza a sus necesidades, motivaciones y expectativas, se puede mencionar un trabajo previo presentado en el XII Congreso Mundial de Profesores de Francés en Quebec, Canadá, investigación de tipo cuantitativo [1]. En los nuevos estudios que aquí reportamos, el objetivo fue escuchar directamente a nuestros alumnos y, su representación de lo que es una lengua extranjera, de cómo se aprende y en particular su sentir sobre la realización de actividades no centradas en el lenguaje mismo.

\section{LAS ACTIVIDADES DE APRENDIZAJE NO CENTRADAS EN EL LENGUAJE}

Algunos teóricos constructivistas de la educación han enfatizado la importancia de enseñar cómo y cuándo usar las

\footnotetext{
${ }^{a}$ Corresponding author, UNAM FES ACATLÁN, https://orcid.org/0000-0002-2654-3367, Email: luenprma2@gmail.com

${ }^{b}$ UNAM FES ACATLÁN, https://orcid.org/0000-0002-3304-5518, Email: anmarusa65@gmail.com
} 
habilidades que se aprenden en la escuela, y no sólo limitarse a los simples conocimientos. Tomando muy en cuenta la idea de Vygotsky de que el aprendizaje es inherentemente social, estos teóricos señalan que la escuela permite la reunión de las personas para construir el conocimiento de manera colaborativa y prepararse para usar esos conocimientos y habilidades, incluyendo las habilidades sociales, en la vida real extra escolar [2]. Sin embargo, las escuelas no siempre mantienen estrecha relación con el mundo y los aprendizajes escolares llegan a estar alejados del mismo. Es cierto que hay limitaciones a las posibilidades de realizar aprendizajes a través de tareas aplicadas a la vida real, pero sin duda el diseño de los programas y en general el diseño instruccional sólo pueden resultar beneficiados de tomar en cuenta las aplicaciones potenciales de la educación. La realización de tareas auténticas o cercanas a lo auténtico favorece el pensamiento crítico y la búsqueda de soluciones a los problemas en situaciones realistas [3]. En convergencia con esta tendencia educativa, las transformaciones mundiales que se han dado en el nuevo siglo, la globalización de la economía y el desarrollo de una sociedad de la información han dado lugar al planteamiento de una educación centrada en las nuevas y complejas competencias orientadas a la acción profesional que requiere el mundo actual [4].

El campo educativo de la enseñanza aprendizaje de lenguas extranjeras no ha sido ajeno a las mencionadas corrientes renovadoras. Christian Puren ha sintetizado diferentes perspectivas en la enseñanza actual de las lenguas culturas, y señala a la acción social a la vez como el nuevo objetivo y el nuevo medio privilegiado para las mismas [5].

La idea de realizar en nuestros cursos de francés actividades no centradas en el lenguaje no es nueva. Hace varios años visitó nuestro centro de idiomas el profesor Claude Germain, de Quebec, quien nos habló de lo que él llama "el francés intensivo". Claude Germain ha establecido en varios sitios del Canadá (como Terranova y el Labrador) cursos de diferentes materias impartidas en francés como lengua extranjera y ha estudiado a fondo los resultados, encontrando que se aprende mejor la lengua segunda cuando el alumno centra su atención en algo diferente de la lengua misma, algo que necesita y que le interesa para diversos fines, y en el curso de esas actividades los alumnos adquieren eficazmente, por inmersión, la capacidad de comunicarse en francés. El aprendizaje es entonces mejor que cuando las actividades se centran únicamente en los aspectos puramente lingüísticos, como la gramática o el léxico. En las palabras del investigador canadiense:

Les récentes recherches sur le cerveau et l'acquisition du langage démontrent que le savoir (telle la connaissance des règles de grammaire) se situe dans notre mémoire déclarative, alors que les habiletés (comme parler sans effort et sans analyser chaque mot, ainsi que nous le faisons dans notre langue maternelle) se situent dans notre mémoire procédurale.
Somme toute, nous n'avons pas besoin d'une grammaire explicite pour apprendre à parler une langue, nous avons besoin d'une grammaire implicite [6].

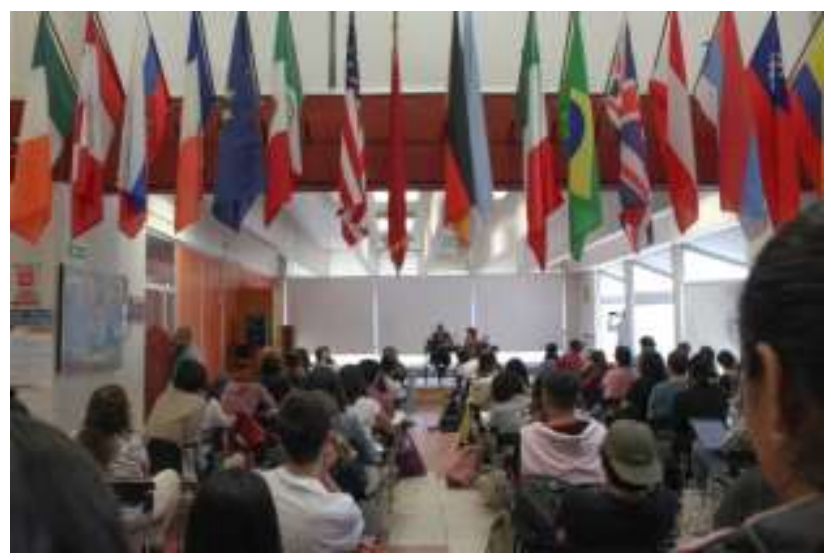

Figura 1. Un ejemplo de actividad centrada en el contenido y no en el lenguaje: conferencia por una escritora de lengua francesa.

Con esa idea, nos propusimos en nuestro Departamento de Francés de la FES Acatlán de la UNAM complementar nuestros cursos ofreciendo a los alumnos actividades variadas, en las que tienen la oportunidad -y la necesidad- de comunicarse en la lengua extranjera. En esto colaboraron todos los profesores de nuestro departamento. Las actividades propuestas fueron las siguientes:

- Conferencias impartidas por profesores de nuestro departamento y por autores francófonos invitados, tanto de manera presencial como en teleconferencia.

- $\quad$ Presentaciones públicas en francés por parte de los alumnos sobre diversos temas de la Francofonía, como escritores, cantantes, gastronomía y otros aspectos culturales de diferentes países.

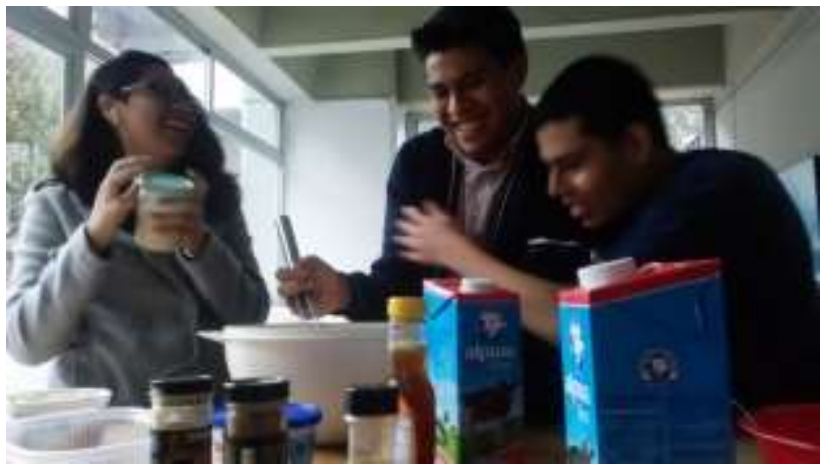

Figura 2. Actividad de cocina realizada en idioma francés en el Centro de Idiomas.

- $\quad$ Canciones y Karaoke

- Cocina

- Juegos 


\section{- Cine \\ - $\quad$ Escenas de teatro}

Cabe también decir que en Acatlán este tipo de actividades también se lleva a cabo en otros departamentos, es decir en otros idiomas, como el italiano, alemán, portugués, japonés, chino, árabe o náhuatl, entre otros.

Al participar en estas actividades, realizadas en francés, los alumnos van necesitando y usando esa gramática implícita de la que habla Claude Germain. La adquisición de la lengua extranjera se propicia en la medida en que se trata de actividades significativas para ellos.

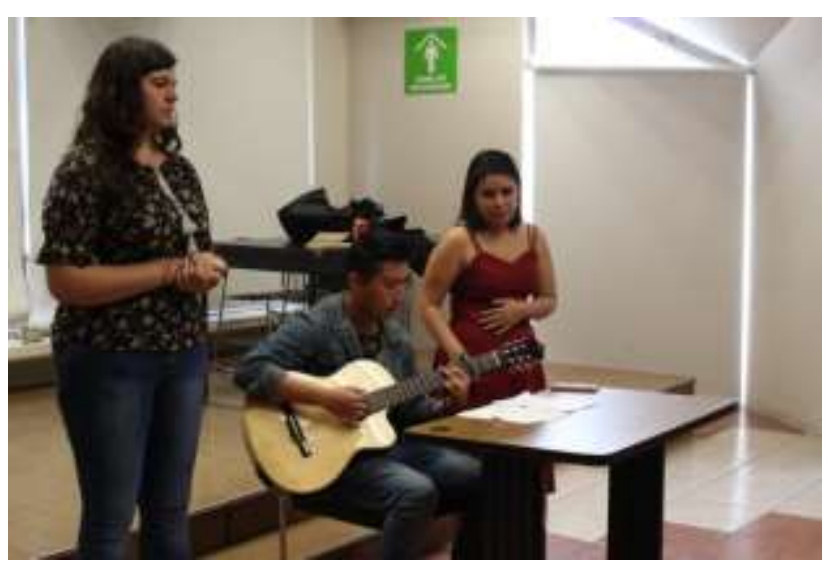

Figura 3. Actividades significativas para los alumnos: interpretación de canciones en lengua extranjera.

Para conocer el resultado de estas actividades, en las que participaron cientos de alumnos, se aplicó un cuestionario de opinión sobre el interés de las actividades y su utilidad para el aprendizaje de la lengua y la cultura extranjeras. El cuestionario completo se muestra en la Figura 4.

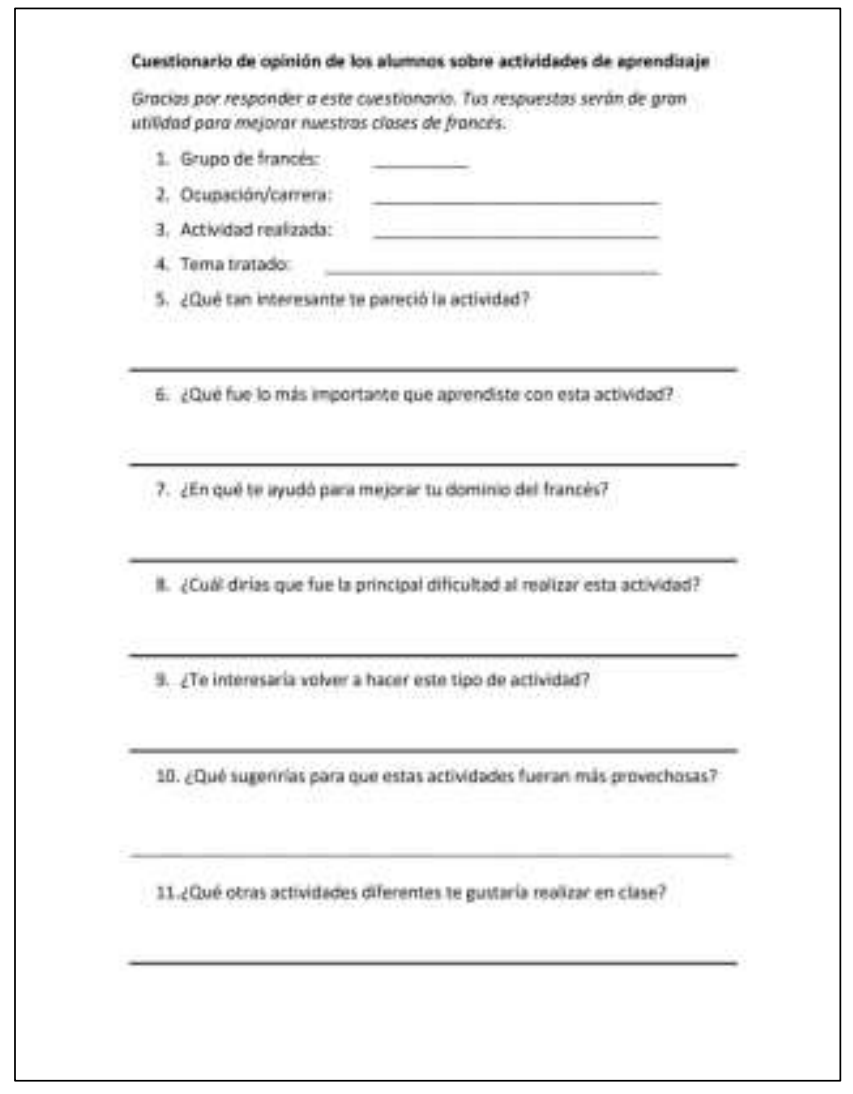

Figura 4. Cuestionario de opinión de los alumnos

Contamos con 78 cuestionarios completos. Cabe subrayar que no se intentó con este instrumento cuantificar el aprovechamiento de los alumnos, sino tener directamente sus opiniones sobre su participación y aprendizaje, así como sus sugerencias para mejorar.

No se presenta aquí el análisis completo de los resultados. Nos limitaremos a mencionar a continuación algunos de los puntos más sobresalientes.

- El interés de los alumnos por las diferentes actividades fue generalizado, aunque no unánime, como es normal. Esto se refleja en las respuestas a la pregunta 11: ¿Qué otras actividades diferentes te gustaría realizar en clase? Todos menos uno respondieron que volverían a participar en este tipo de actividades, ya fuera como asistentes o como participantes activos. Las actividades más gustadas son los juegos (25 menciones), conversación y debates, en particular con hablantes nativos (25 menciones), el cine (15 menciones), la música incluyendo karaoke (14 menciones) y la cocina (12 menciones).

- Las respuestas a la pregunta 6 ¿Qué fue lo más importante que aprendiste con esta actividad? se refieren en su mayoría a aspectos formativos y culturales, como aprender a hablar en público y 
perder el miedo, aprender a trabajar en equipo, investigar y aprender a hacer una presentación en público, o incluso descubrir, preparar y compartir platillos de la Francofonía, no sólo de países europeos, sino asimismo por ejemplo del Canadá o de África. Llama la atención que en general los alumnos perciben estas actividades más como parte de su formación general que como simples actividades de aprendizaje de la lengua extranjera. Sin embargo, aunque las actividades no se centraban en el lenguaje, un número significativo de estudiantes mencionaron entre lo más importante

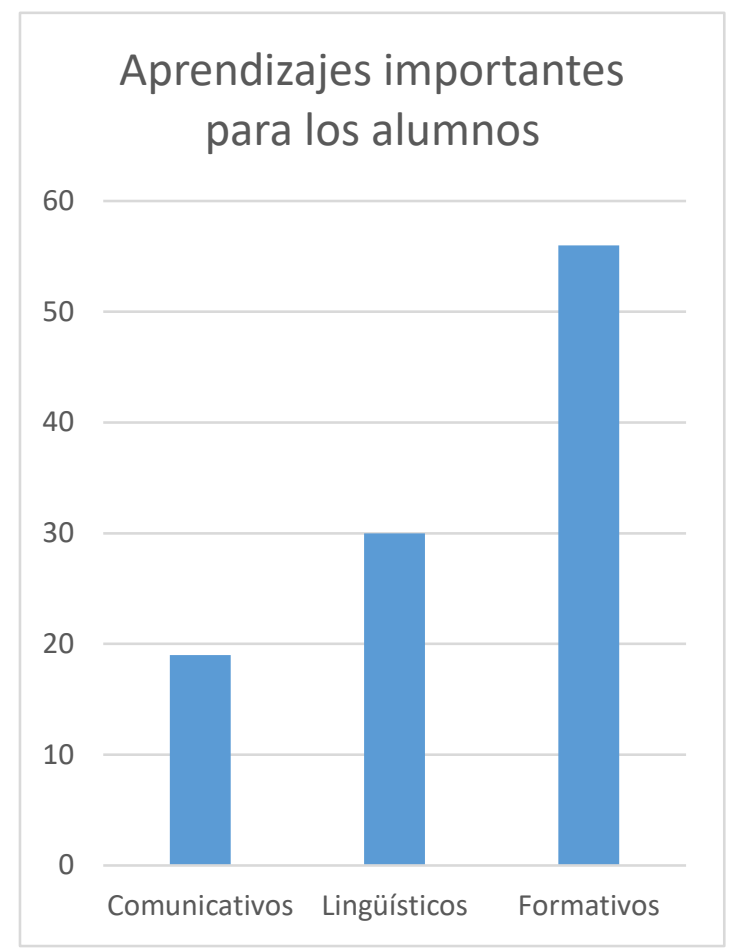

que habían aprendido aspectos lingüísticos y comunicativos (Gráfica 1).

Gráfica 1. Aprendizajes importantes para los alumnos a partir de las actividades realizadas

- A la pregunta 7 ¿En qué te ayudó para mejorar tus habilidades con el idioma francés? la mayoría de las respuestas se refirieron al mejoramiento de la comprensión auditiva y de la pronunciación, y después se mencionaron el aprendizaje de léxico y expresiones del idioma (Gráfica 2).

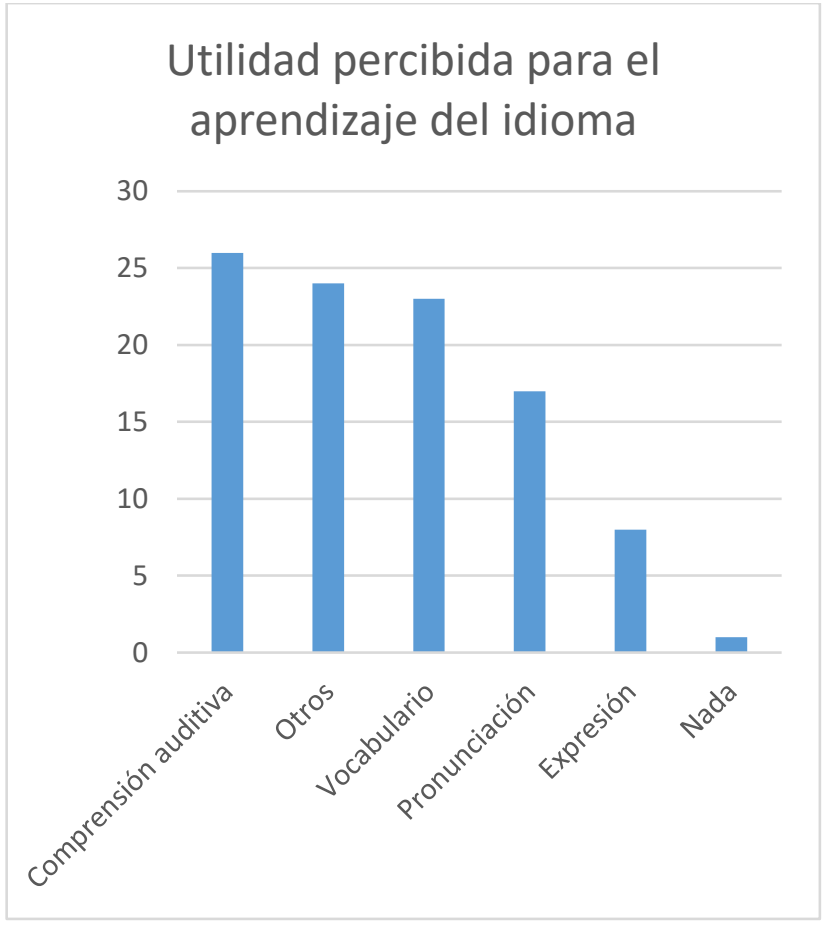

Gráfica 2. Utilidad percibida por los alumnos para el aprendizaje del idioma con las diferentes actividades.

- Por último, cuando se pidió a los estudiantes que dieran sus sugerencias para hacer más provechosas estas actividades, muchas respuestas se refirieron a aumentar la variedad de las tareas propuestas, así como a mejorar la organización y difusión de las mismas, a aumentar su frecuencia y su disponibilidad en diferentes horarios (Gráfica 3).

\section{Actividades preferidas por los alumnos}

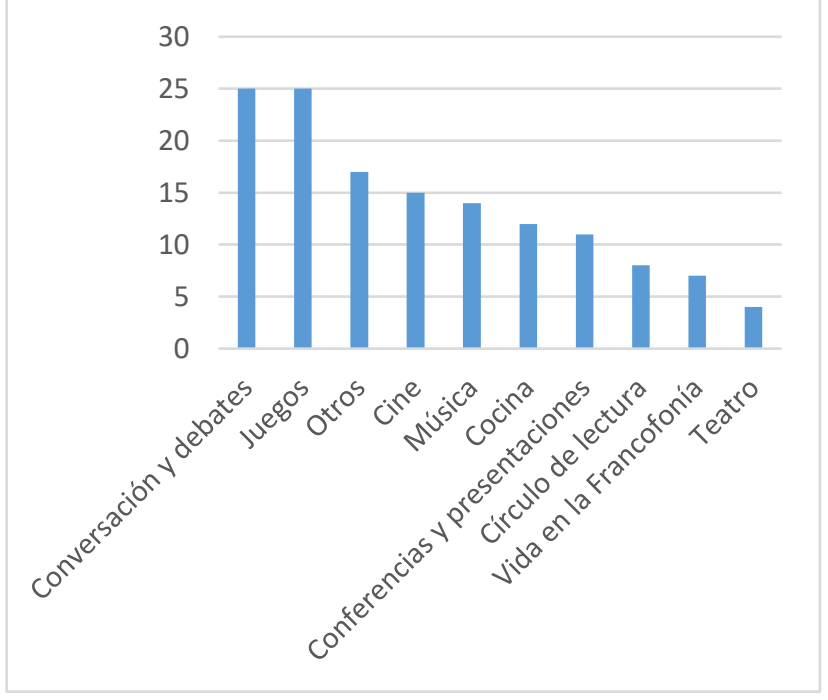

Gráfica 3. Actividades preferidas por los alumnos para complementar y aplicar sus aprendizajes. 


\section{LA PERCEPCIÓN DE LOS ALUMNOS SOBRE LA LENGUA EXTRANJERA Y SU APRENDIZAJE}

La idea de entrevistar a nuestros alumnos para conocer en profundidad su percepción de lo que es una lengua extranjera, cómo y por qué se aprende, tiene por objetivo liberarse del sesgo tradicional impuesto por el profesor sobre las decisiones educativas fundamentales referentes a lo que el alumno necesita y debe aprender, sobre lo que le será útil en la vida y en su carrera profesional, así como sobre las maneras de adquirir los conocimientos y habilidades en una lengua extranjera.

Con demasiada frecuencia, los profesores deciden que los estudiantes deben aprender idiomas para leer textos especializados en lengua extranjera, para obtener certificaciones según el marco europeo de referencia, para estudiar posgrados en el extranjero, para competir por mejores trabajos... Cabe cuestionar en qué medida esas decisiones se basan en prejuicios y estereotipos, y en qué medida se conoce realmente a los alumnos, sus motivaciones, sus intereses, sus proyectos, sus estilos de aprendizaje o sus dificultades.

Se grabaron horas de entrevistas en las que se dejó discurrir libremente a los alumnos sobre el camino que los llevó a aprender idiomas, sobre lo que ellos conciben como una lengua extranjera asociada a una cultura, sobre lo que les gusta realizar con ella, e incluso sobre las características que aprecian en un buen profesor. Los alumnos pudieron escoger el idioma en que se realizaba la entrevista, y el contrato de entrevista deja en custodia del investigador las grabaciones originales, solo permitiéndole publicar la transcripción de fragmentos anónimos para propósitos académicos.

Varios aspectos comunes que destacan claramente de entre la diversidad de individualidades que se integran en la vida y el trabajo de un centro de idiomas como el de Acatlán, donde cada semestre varios miles de alumnos vienen para aprender algunos de los quince idiomas que se imparten.

En primer lugar, de las entrevistas resulta muy claro que los alumnos siguen aprendiendo idiomas porque les gusta. Saben que los idiomas les serán útiles en su vida profesional, y además en la mayoría de las carreras del campus es obligatorio acreditar el conocimiento de una lengua extranjera. (aunque según los alumnos, para sus estudios en las diferentes materias de sus carreras casi no se les dan lecturas en lenguas extranjeras). Sin embargo, muchos de ellos aprenden dos, tres, cuatro idiomas extranjeros, y cuando se les pregunta ¿por qué estudias francés? o japonés, o árabe o ruso, a menudo responden "porque me gusta". Cuando se les pregunta ¿Qué te gusta del idioma? y ¿Qué te gustaría hacer con el idioma? ya se entra de lleno en el terreno de la individualidad. Esto implica que nuestros programas y nuestras actividades didácticas deben ofrecer la apertura y la flexibilidad para que dentro del trabajo grupal se puedan manifestar las individualidades.

Hay que preguntar a los alumnos y darles libertad. En palabras de una alumna respondiendo a la pregunta ¿Qué es para ti un idioma?:

Un idioma es una probadita de otra cultura, es que te puedas meter tantito a ver [...] que te puedas imaginar cómo piensan en este caso los francoparlantes, es que te metas a la cultura y que te despejes, o sea que dejes de pensar en tu idioma, en tu cultura, para que pienses en otra, pero en tu misma cabeza.

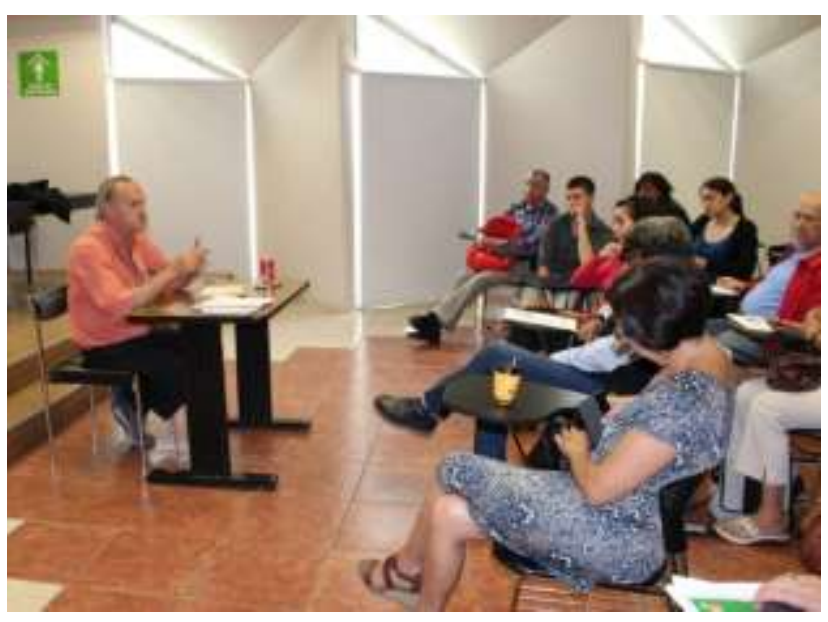

Figura 5. Un idioma es una probadita de otra cultura.

En segundo lugar, se pone de manifiesto que los alumnos tienen habilidades metacognitivas bien desarrollada y son conscientes de lo que ya saben y dominan, pero también de cuáles son sus puntos débiles y qué actividades esperan realizar en sus cursos y con el apoyo del profesor, para mejorar en sus habilidades en lengua extranjera. En respuesta a la pregunta ¿Cómo ha cambiado tu percepción de lo que representa aprender un idioma? responde una alumna:

Antes era por gusto, porque yo quería saber desenvolverme si yo me iba a algún otro lugar. Yo me quería ir a trabajar a algún otro lugar, y bueno, o a viajar. Y ahorita ya lo veo más como un plus y una oportunidad en mi vida, y por ejemplo en mi vida laboral (K. Guerrero, comunicación personal, 3 de septiembre 2019).

Destaca que usan la tecnología en mayor o menor medida para aprender y para vivir, característica que necesariamente debe tomarse en cuenta en el proceso educativo. En el campo de las lenguas, como en general en la educación actual, la tecnología, las TICs, son el presente y el futuro. En la UNAM los estudiantes usan la tecnología para inscribirse a los cursos, para coordinarse en las tareas, para buscar información, libros, ejercicios, usar diccionarios y traductores, podcasts, canciones, series televisivas, redes en lenguas extranjeras, para saber si el profesor se enfermó y no va a llegar a dar 
clase. Las aulas virtuales y los exámenes en línea, que eran una opción más hasta tiempos recientes, se han convertido con la crisis sanitaria del año 2020 en una herramienta esencial para los procesos educativos. Esto conlleva también para los profesores la necesidad de perfeccionarse y actualizarse en el manejo educativo de las TICs, las cuales deben integrarse dentro de proyectos educativos adecuados y estructurados.

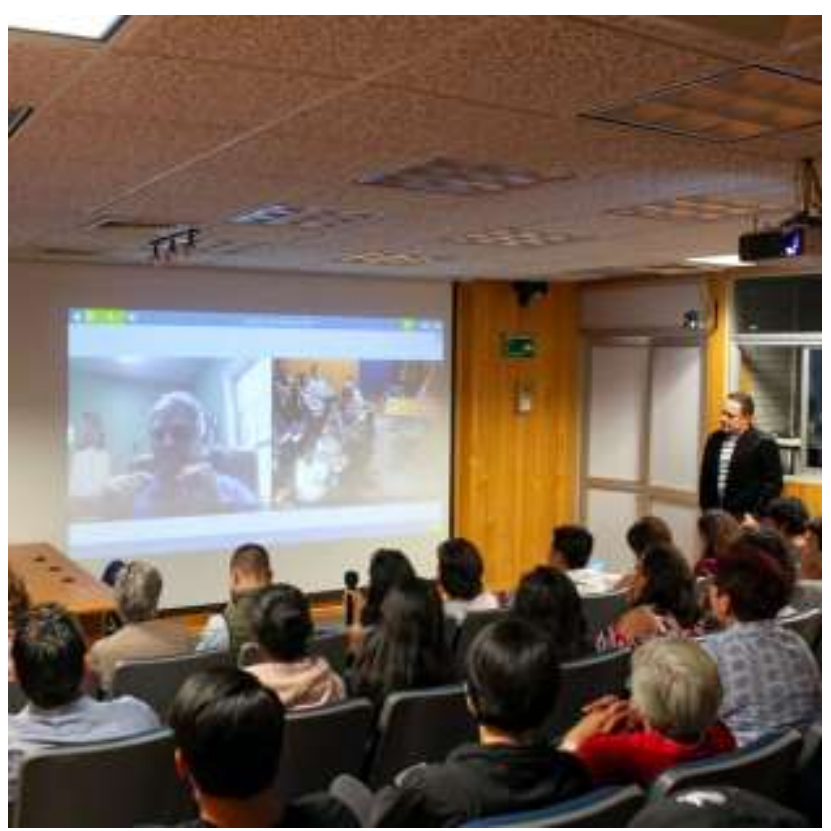

Figura 6. Nuevas tecnologías: Teleconferencia en lengua extranjera

El tercer punto destacado en nuestras entrevistas es que los estudiantes universitarios muestran poseer valores de compromiso con su entorno social y ecológico, aprecian la solidaridad, el respeto y la no discriminación. La Universidad debe basar sus grandes proyectos y sus actividades educativas en una coherencia con dichos valores fundamentales.

\section{CONCLUSIONES Y RECOMENDACIONES}

La primera constatación que se puede hacer a partir de nuestros estudios es confirmar que la investigación aplicada a nuestra situación educativa concreta nos da la oportunidad de mejorar nuestra práctica como docentes. A pesar de sus alcances modestos, estos trabajos nos han permitido conocer con mayor precisión los intereses de nuestros alumnos y su percepción de su propio aprendizaje. Si esta información se incorpora subsecuentemente al diseño de nuestros programas y en general a la planeación de nuestras actividades pedagógicas, es de esperarse que todo el proceso en esta área de nuestra institución educativa, en este caso en la enseñanza de idiomas, pueda verse muy beneficiado. Queda claro en consecuencia que un trabajo de esta naturaleza constituye sólo un paso necesario que debe ser seguido de la aplicación a nuestra acción educativa. Resulta asimismo evidente que el conocer de primera mano a nuestros alumnos es indispensable si se pretende realmente centrar el proceso educativo en ellos mismos. Pese a que el hablar de la educación centrada en el alumno se ha vuelto casi un lugar común, a menudo son los profesores y los diseñadores educativos quienes toman las decisiones sobre las necesidades, intereses y motivaciones de los estudiantes, con toda la distorsión que esto puede implicar.

Se ha mencionado el alcance modesto de este estudio. Una limitación importante, que apunta sin embargo hacia investigaciones y avances ulteriores, es el hecho de que estos estudios sólo se refirieron a la opinión y percepción de los alumnos, incluyendo en ello a su propio aprendizaje. Sin embargo, no se realizaron pruebas objetivas del progreso en las habilidades tras las actividades realizadas. Un mejor conocimiento de la contribución de éstas al aprendizaje de una lengua extranjera requerirá estudios cuantitativos de las habilidades y competencias lingüísticas antes y después de las actividades propuestas. En nuestro contexto particular, esto implica una labor para contribuir a una cultura de la investigación educativa como parte del trabajo institucional, pues la colaboración tanto de las autoridades como del conjunto de los docentes es indispensable para le ejecución de investigaciones con muestras estadísticas aleatorias válidas y grupos de control. La otra gran vertiente para proseguir este trabajo es la comparación con otros contextos más o menos semejantes, especialmente en nuestro país. La esencia misma de la difusión académica de estas experiencias, ya sea en congresos o publicaciones, es propiciar una colaboración más amplia en la búsqueda de soluciones educativas, en beneficio de nuestra propia institución y, es de esperarse, de otras instituciones que podrían participar en un esfuerzo educativo conjunto comprometido socialmente. Aunque nuestros estudios no son de naturaleza teórica, la mencionada colaboración académica implica también el enriquecimiento de los indispensables fundamentos teóricos, incluso en su caso gracias a la confrontación de puntos de vista contradictorios.

Concretamente, de las encuestas y entrevistas realizadas se concluye que deben incluirse actividades complementarias en los cursos de idiomas, en las cuales los estudiantes pongan en práctica las habilidades adquiridas mediante la realización de "tareas", en el sentido de realizaciones auténticas de comunicación y acción. Esto último debe entenderse de una manera amplia, para incluir desde la simple asistencia a una conferencia o espectáculo, hasta la ejecución de proyectos colaborativos de tipo ecológico, cultural, social, etc.

Los acontecimientos globales del año 2020 han dejado definitivamente en claro que la educación actual en todos sus aspectos, como en el ejemplo particular de los idiomas, objeto de este estudio, debe incorporar las nuevas tecnologías que constituyen una parte esencial del mundo contemporáneo y estar abierta a los desarrollos futuros para mantenerse a la vanguardia y a la altura de los retos a los que se enfrenta. 
Las actividades educativas deben ofrecer la flexibilidad y la libertad para adaptarse a las diversas motivaciones y estilos de aprendizaje de los estudiantes. Estas características no deben darse únicamente en eventos más o menos aislados agregados a las actividades destinadas a cumplir los programas. En momentos en que los acontecimientos sociales y políticos hacen indispensable el replanteamiento del conjunto del curriculum educativo nacional y la actualización de los profesores, los programas deben dar el máximo espacio posible de flexibilidad y libertad para los actores del proceso. Asimismo, deben ser consistentes con los valores de una universidad comprometida con la sociedad en la que se haya inserta. La participación conjunta de autoridades, profesores y estudiantes es imprescindible para el logro de los grandes objetivos educativos requeridos por la complejidad del mundo actual.

\section{REFERENCIAS}

[1] Prieto Marín, L.E. y Rueda Salazar, A.M. (2008). Connaître les représentations des élèves pour créer du matériel multimédia sur mesure. En: Faire vivre les identités francophones, Actas del XII Congreso mundial de la FIPF, Quebec, 21-25 julio 2008 (p.10291036). Quebec : FIPF.

[2] Vygotsky, L.S. (1978) Mind in society: The development of higher mental process. Cambridge, MA : Harvard University Press.

[3] Lave, J. y Wenger, E. (1991). Situated learning : Legitimate peripheral participation. Cambridge: Cambridge University Press.

[4] Echeverría, B. (2002). Gestión de la competencia de acción profesional. Revista de investigación educativa, 20(1), 7-43.

[5] Puren, C. (2009). Variations sur la perspective de l'agir social en didactique des langues-cultures étrangères. En Rosen, E. (Ed.) La perspective actionnelle et l'approche par les tâches en classe de langue (pp. 154-167). Paris : CLE International.

[6] Germain, C. y Netten, J. (2002). Fondemants d'uner approche transdisciplinaire en FLE/FL2 : Le français intensif au Canada. Cahiers du français contemporain. 